\title{
Chromosomal instability and cancer: a complex relationship with therapeutic potential
}

\author{
Samuel F. Bakhoum and Duane A. Compton
}

Department of Biochemistry and Norris Cotton Cancer Center, Dartmouth Medical School, Hanover, New Hampshire, USA.

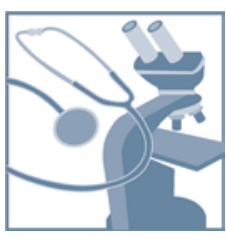

Chromosomal instability (CIN) is a hallmark of human neoplasms. Despite its widespread prevalence, knowledge of the mechanisms and contributions of CIN in cancer has been elusive. It is now evident that the role of CIN in tumor initiation and growth is more complex than previously thought. Furthermore, distinguishing CIN, which consists of elevated rates of chromosome missegregation, from aneuploidy, which is a state of abnormal chromosome number, is crucial to understanding their respective contributions in cancer. Collectively, experimental evidence suggests that CIN enables tumor adaptation by allowing tumors to constantly sample the aneuploid fitness landscape. This complex relationship, together with the potential to pharmacologically influence chromosome missegregation frequencies in cancer cells, offers previously unrecognized means to limit tumor growth and its response to therapy.

\section{Introduction}

Adaptation is a hallmark of human neoplasms, and the ability of tumors to adapt to external pressures stems from tumor cell heterogeneity (1). Mechanisms responsible for this heterogeneity include DNA mutation, chromosomal translocation, and gene amplification. However, an additional pathway leading to tumor cell heterogeneity involves elevated frequencies of whole-chromosome missegregation - otherwise known as chromosomal instability $(\mathrm{CIN})(2,3)$. CIN is a hallmark of most solid tumors (4), and it has long been postulated that chromosome missegregation is an important mechanism of tumor adaptation (1, 3). However, it was not until 1997, when Lengauer and Vogelstein directly demonstrated evidence of persistent chromosome missegregation in cancer cell lines, that work exploring the role of CIN in tumorigenesis began (2).

A direct consequence of CIN is aneuploidy (5), which has been implicated in tumorigenesis for decades (6). Many hematopoietic malignancies were found to be clonally aneuploid, with an identifiable karyotype that carries a prognostic value (7-10). Furthermore, individuals with constitutional global aneuploidy, such as those with Down syndrome, mosaic variegated aneuploidy, and Turner syndrome, are found to exhibit increased rates of malignancies (11-16). Whereas aneuploidy and CIN are interrelated, they are not synonymous, and confusion in the literature arises from using both terms interchangeably (3). In many cases, aneuploidy can be present independently of CIN by resulting from a single event of chromosome missegregation, with subsequent clonal expansion of the aneuploid karyotype. In such cases, tumors are homogeneously aneuploid (17). CIN, on the other hand, typically yields a heterogeneously aneuploid tumor cell population that has the ability undergo selective evolution, as is required for processes such as metastasis and resistance to therapy. Work now shows that aneuploidy and CIN may have independent contributions to tumor evolution and growth, even while coexisting throughout the tumor's lifetime.

\section{Mechanisms of CIN}

Chromosome segregation during mitosis requires the proper attachment of chromosomes to microtubules at kinetochores (18).

Conflict of interest: The authors have declared that no conflict of interest exists. Citation for this article: J Clin Invest. 2012;122(4):1138-1143. doi:10.1172/JCI59954.
Kinetochores constitute the macromolecular sites at the centromeric regions of chromosomes that regulate their attachments to microtubules (19). Dozens of gene products are involved in ensuring chromosome segregation fidelity, and therefore it is not surprising that defects in multiple mechanisms that lead to errors in chromosome segregation appear in cancer (20). These include faulty sister chromatid cohesion (21), defective centrosome duplication (22-24), telomere dysfunction (25), hyperactive or hypoactive spindle assembly checkpoint (SAC) (26-28), and overly stable attachments of microtubules to chromosomes (29, 30). Most of these mechanisms converge to produce lagging chromosomes during anaphase (5, $24,29,30)$. Chromosomes lag due to their erroneous attachment to microtubules emanating from opposite spindle poles $(31,32)$. This results in their presence at the mitotic spindle midzone during anaphase, separated from the properly segregating chromosome masses (Figure 1). In fact, direct live-cell imaging shows that most chromosomally unstable cancer cell lines exhibit elevated frequencies of lagging chromosomes, while stable diploid cells do not (5). Furthermore, altered frequencies of lagging chromosomes were shown to directly correlate with altered chromosome missegregation when observing individual cell division events (29).

The process of chromosome segregation begins at nuclear envelope breakdown when mitotic chromosomes initiate contact with microtubules of the nascent spindle (33). The interaction of microtubules with the chromosomes is stochastic, yet sister chromatids must become attached to opposite spindle poles prior to the onset of anaphase if chromosomes are to properly segregate (refs. 33-35 and Figure 1A). Further complicating the segregation process is the ability of each chromosome to stably attach to 20 to 25 microtubules at kinetochores (36), and, in many instances, individual kinetochores are attached to microtubules emanating from opposite spindle poles. This erroneous attachment must be corrected prior to anaphase onset to avoid the formation of lagging chromosomes $(31,32)$ (Figure 1A). The correction of attachment errors is made possible by the dynamic behavior of microtubules (37), whereby they constantly attach and detach from chromosomes at measurable rates on the order of minutes $(30,38)$. It was shown that simply increasing the average duration of attachment of microtubules to chromosomes - by depleting microtubule destabilizers at the kinetochore - leads to the persistence of attachment 
A

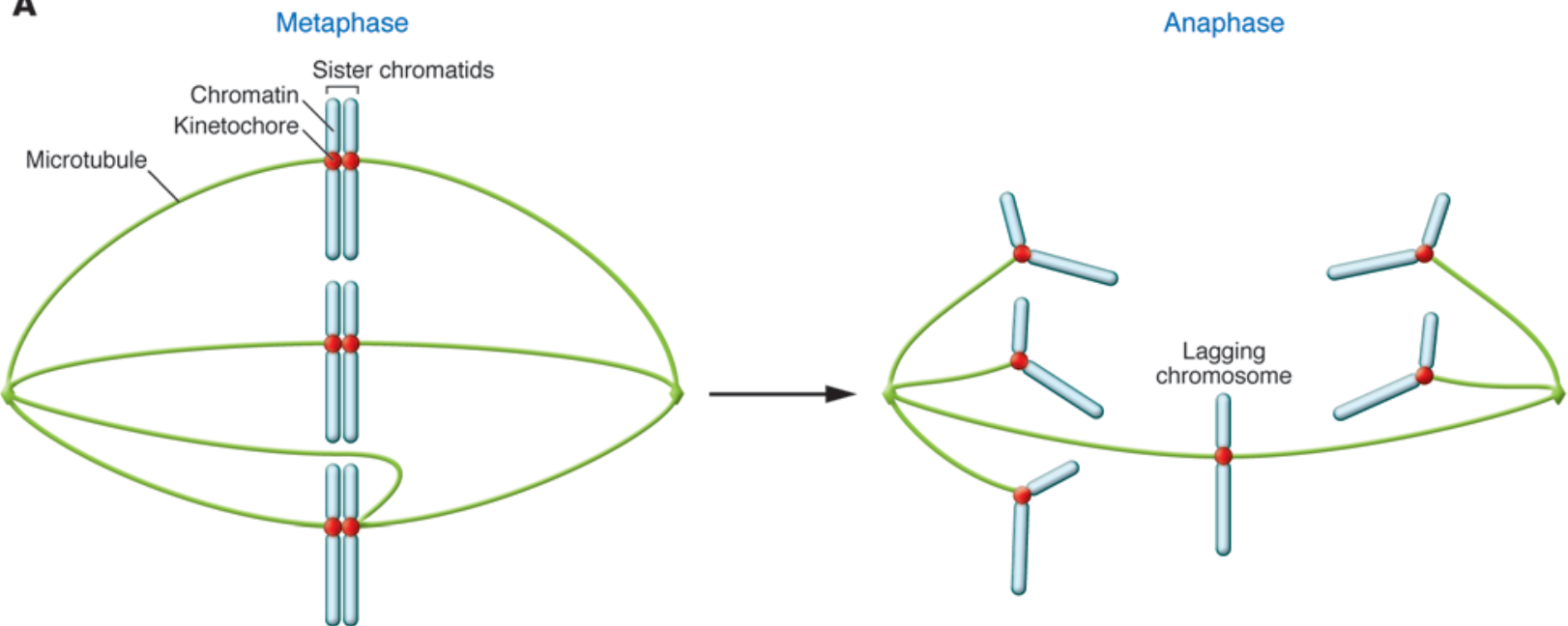

B
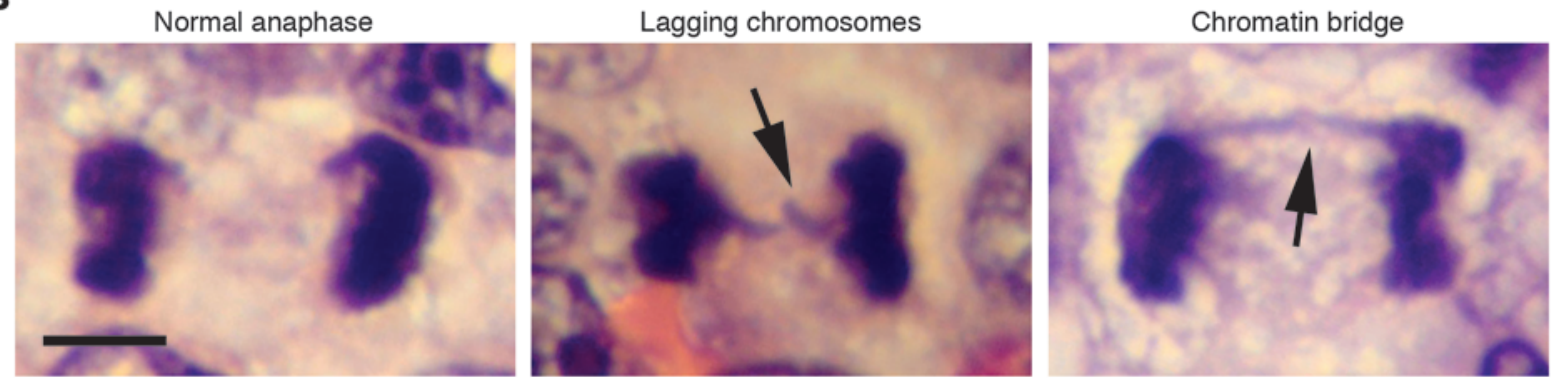

Figure 1

Lagging chromosomes as markers of CIN. (A) Schematic diagram of a mitotic spindle at metaphase and at anaphase, depicting normally attached "bi-oriented" chromosomes as well as 1 lagging chromosome, which is improperly attached simultaneously to both spindle poles. Upon anaphase onset, this improper attachment can lead to lagging chromosomes. Microtubules, kinetochores, and chromatin are depicted in green, red, and blue, respectively. (B) H\&E-stained cells undergoing anaphase from tumor biopsies of patients with DLBCL, exhibiting lagging chromosomes (arrow, middle) as well as chromatin bridges (arrow, right), which frequently result from lagging chromosomes. Scale bar: $5 \mu \mathrm{m}$.

errors and resultant chromosome missegregation, while decreasing the duration of attachments suppressed CIN in cancer cells with high rates of missegregation $(29,30)$. Furthermore, work linking centrosome duplication to CIN demonstrated that the presence of extra centrosomes increases the rate of formation of erroneous attachments to a point that overwhelms the correction rates achieved by microtubule dynamics $(23,24)$. This happens because multipolar spindles coalesce their supernumerary poles and become bipolar spindles that contain numerous errors in the attachment of microtubules to chromosomes, ultimately leading to the presence of lagging chromosomes during anaphase $(23,24,39)$. Finally, it was shown that both hyperactive as well as hypoactive SAC signaling can directly lead to chromosome missegregation $(27,28,40-42)$.

The SAC is a signaling cascade that functions during cell division to delay the onset of anaphase until all chromosomes have aligned along the metaphase plate and each sister chromatid is attached to a unique spindle pole (43). SAC signaling originates at kinetochores, and it is tightly coupled to the occupancy of attached microtubules; it is evident that stable kinetochore-microtubule attachments are necessary to allow for anaphase onset (44). In the case of reduced SAC signaling, cells undergo anaphase prematurely before the erroneous attachments of microtubules to chromosomes are corrected. However, in examples of hyperactive
SAC signaling, the mechanism resulting in chromosome missegregation is not entirely understood. It is possible that persistent SAC signaling can lead to metaphase arrest and the formation of tetraploid cells (45), which have been shown to confer increased tumorigenic potential and higher tolerance of and susceptibility for chromosome missegregation (45). Alternatively, some proteins involved in SAC signaling could also be involved in the regulation of microtubule attachments to chromosomes, and therefore a hyperactive SAC would also indirectly increase the stability of erroneous attachments leading to chromosome missegregation (42).

Finally, recent work described a process termed chromothripsis, in which massive chromosomal rearrangements occur in cancer cells when single chromosomes are fragmented, followed by the cellular attempt for repair (46). Typically, this repair is far from perfect, and many chromosomal regions are lost. Although this is not considered whole-chromosome missegregation, repeated loss of chromosomal segments can lead to the same effect as aneuploidy. It is notable that chromothripsis is estimated to occur in $2 \%$ to $3 \%$ of all cancers and $25 \%$ of bone cancers $(46,47)$.

The majority of the work involved in identifying these mechanisms was performed in cancer cell lines or mouse models. In many cases, the starting material consisted of normal chromosomally stable diploid cells, which were subsequently made aneuploid and chromosomally unstable. Thus, it remains to be seen 


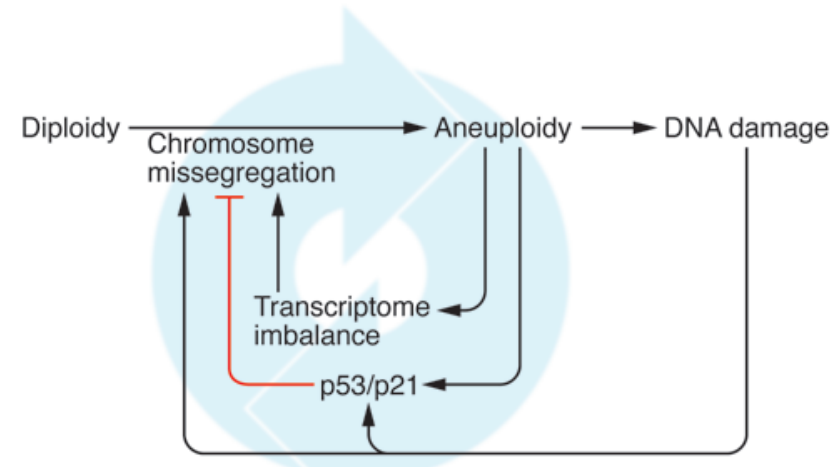

Figure 2

The relationship between ploidy and CIN in the context of tumor initiation and growth. Frequent chromosome missegregation events lead to aneuploidy, which in turn leads to transcriptome imbalance and additional chromosome missegregation as well as DNA damage. Aneuploidy brought about by chromosome missegregation also triggers the p53/p21 tumor suppressor pathway, which in turn limits further proliferation of aneuploid cells. The circular arrow depicts the proposed self-propagating nature of $\mathrm{CIN}$ in tumor cells.

whether these mechanisms of chromosome loss indeed take place in human cancers, and strong clinically derived evidence is still lacking. However, it was recently shown that lagging chromosomes are prevalent in fixed tissues taken from patients diagnosed with diffuse large $B$ cell lymphoma (DLBCL) (Figure 1B) and that the frequencies of lagging chromosomes significantly correlated with tumor prognosis and overall survival (48). Collectively, data from cancer cell lines as well as fixed tumor samples suggest that lagging chromosomes may be the major mechanism leading to chromosome missegregation in cancer.

\section{CIN in tumor initiation and growth}

The role of chromosomal imbalance in tumorigenesis has been appreciated for over a century, since Théodore Bovéri observed aneuploid karyotypes in cancer cells (6). It is now evident that more than $70 \%$ of common solid neoplasms are aneuploid $(4,49)$, and, in many instances, the onset of heterogeneous aneuploidy correlates with the aggressiveness and stage of the tumor (40,50-54). As a result, it has been a long-standing assumption that chromosome missegregation is a driver of tumor initiation and growth.

Experimental evidence has implicated CIN in tumor initiation (55). In one study, inducing chromosome missegregation in mice by perturbing microtubule attachments to chromosomes led to the spontaneous formation of lymphomas and lung tumors (56). Furthermore, overexpressing the kinetochore-microtubule stabilizing protein hec1 in mice induced the formation of lung adenomas, liver carcinomas, hemangiosarcomas, and islet cell hyperplasias (28). It was also shown that increasing SAC signaling by overexpressing Mad2 led to CIN, and this was required for the development of anaplastic tumors in a mouse model of mammary adenocarcinoma (57). Interestingly, the creation of tetraploid cells, which are more prone to chromosome missegregation, was also shown to increase tumor formation (45). It is important to note that, in many of these studies, an additional genetic alteration was required for tumorigenesis, such as the deletion of the $p 53, p 21$, or $p 19^{\text {Arf }}$ tumor suppressor genes $(45,56)$. Accordingly, it was shown that disruption of the p53/p21 pathway was required for the tolerance of aneuploid karyotypes, and inducing chromosome missegregation in cells with an intact p53 signaling pathway led to durable cell cycle arrest (ref. 58 and Figure 2). Together, these findings raise the question of the precise role of CIN in tumor evolution. The dependence of CIN on defective p53 signaling suggests that elevated frequencies of chromosome missegregation likely occur at an intermediate stage in tumor evolution, and the prevalence of chromosome missegregation during early tumorigenesis in humans remains unclear. It is theoretically possible that CIN may be sufficient to induce tumor formation by directly inactivating the p53 signaling pathway. Experiments in mammalian cells show that the number of chromosome copies proportionately correlates with the level of mRNA in cells $(59,60)$; given that individual human chromosomes encode an estimated thousand genes each, the presence or absence of single chromosomes can have a dramatic effect on whole-cell gene expression. The resulting imbalance has the potential to deregulate cell cycle proteins, tumor suppressors, and oncogenes. Indeed, in mice that were heterozygous for tumor suppressor genes $p 53$ and APC, inducing CIN led to the loss of heterozygosity and the development of thymic lymphomas and colonic tumors, respectively (61). Finally, recent work shows that the process of chromosome missegregation can directly lead to DNA damage and translocation (62). In this study, Janssen et al. showed that lagging chromosomes are frequently damaged during cytokinesis, triggering a DNA double-stranded break response involving the ATM, Chk2, and p53 proteins.

Experimental mouse models demonstrate that inducing CIN also leads to increases in tumor growth rate. In a mouse model in which lung tumor growth was driven with the Kras oncogene, withdrawing the oncogene did not prevent tumor relapse when CIN was induced by overexpressing the SAC protein, Mad2 (27). This suggests that CIN could be an autonomous driver in the latter stages of tumor growth, overriding dependence on the initial tumorigenic signals. These findings corroborate longitudinal observations of human neoplasms, in which the rate of aneuploidy and karyotypic abnormalities (and by inference, CIN) increases with increasing tumor grade and invasiveness (40, 50-54).

The simple role of CIN as a promoter of tumor initiation and growth has been recently challenged by surprising experimental findings showing that chromosome missegregation and aneuploidy can also act to inhibit tumorigenesis (63). In some of these experimental settings, the effect of chromosome missegregation on tumorigenesis may be tissue specific. For instance, inducing CIN appears to have an antitumorigenic effect in the liver (56). This is particularly interesting given that the liver is largely a polyploid organ, with potentially increased basal rates of chromosome missegregation. Thus, it is possible that further increasing missegregation levels can be physiologically intolerable and reduce cellular fitness. This postulate is supported by experiments in cancerderived human cell lines showing that increasing chromosome missegregation beyond a certain level can be cytotoxic (64). In murine syngeneic models of lung cancer, targeting cyclin E led to multipolar cell division combined with massive chromosome missegregation, which had a significant antineoplastic effect (65). In another mouse model, protein levels of the SAC signaling protein Bub1 are reduced to generate CIN. Bub1 is involved in the SAC signaling as well as regulation of microtubule attach- 
ments to chromosomes. While Bub1 heterozygosity increased the number of colonic tumors in the susceptible $A P C^{\text {min/+ }}$ strain, $B u b 1$ heterozygous mice had lower propensity for small intestinal tumors compared with that of wild-type mice (66). Along these lines, computational work predicts that a chromosome missegregation rate between $10^{-3}$ and $10^{-2}$ per chromosome is optimal for cellular fitness (67). These rates correspond to single chromosome missegregation events every 2 to 20 divisions, which are also in line with observations in human cancer cell lines and tumor samples in which the frequencies of cells exhibiting chromosome missegregation ranged from $10 \%$ to $60 \%(5,48)$. In its essence, this permissible boundary of chromosome missegregation is not a novel concept given that CIN is a cellular agent of change. The same idea is proposed for the level of DNA mutation that permits the fitness of a population (68); at these optimal rates, adaptation is made possible, but the ability to change is sufficiently limited to preserve acquired beneficial traits.

\section{CIN and the aneuploid fitness landscape}

A considerable challenge in the field stems from the salient need to separate the individual contributions of aneuploidy from those of CIN. This might not be a simple task, given that chromosome missegregation leads to aneuploidy, and it is technically difficult to experimentally obtain homogeneously aneuploid cells that do not exhibit elevated frequencies of chromosome missegregation. Furthermore, it is not feasible to directly observe chromosome missegregation events in human tumors in real time. As a consequence, aneuploidy has frequently been used as a surrogate marker for CIN $(27,56,69,70)$. For instance, studies searching for gene expression signatures for CIN in human cancers instead used expression profiles common to aneuploid cells, under the assumption that these cells are also likely to exhibit CIN (71). At the same time, in most experiments performed in mouse models, chromosomally stable diploid cells are transformed into both aneuploid and chromosomally unstable cells, making it challenging to determine whether aneuploidy or CIN are the drivers of tumorigenesis. The complex relationship between CIN and tumor initiation and growth may be better understood when a clear distinction between CIN and aneuploidy is kept in mind. Given the plethora of genes on each chromosome, the possible combinations of gene transcript imbalance created by aneuploid karyotypes are incredibly large. In addition, not all aneuploid karyotypes are favorable for either tumor initiation or growth, just as mutations can be beneficial or deleterious to tumor cells. We term the ever-changing cellular fitness over the wide range of possible aneuploid karyotypes the aneuploid fitness landscape, in which both favorable and unfavorable chromosomal combinations exist. This concept is supported by experimental evidence in yeast showing that aneuploidy leads to quantitative proteome changes and phenotypic variations; however, this depended on the environmental condition, and not all aneuploid karyotypes behaved similarly (72). Since cancer is an evolutionary disease, it seems likely that tumor cells with specific chromosomal combinations - such as loss of heterozygosity of tumor suppressor genes $(61,67)$ - would experience a growth advantage. In this way, the tumor would be sampling the aneuploid fitness landscape to select for chromosomal combinations that confer survival benefit. Meanwhile, the dynamic nature of selection pressures on tumors is likely to alter the relative fitness of a given karyotype, thus it is expected that a certain karyotype, favorable under normal growth conditions, may not be growth-promoting when the tumor is challenged with chemotherapy. This fits with the experimental evidence that chromosome missegregation must fall within physiologically permissible boundaries, whereby missegregation levels that are too high can drive cells quickly out of favorable karyotypes (64), whereas excessively low missegregation frequencies can hamper the tumor's ability to continuously sample the aneuploid fitness landscape that is slowly but constantly shifting.

\section{CIN and tumor prognosis}

Even though the relationship between aneuploidy and tumor prognosis has been established for many decades, evidence for the selective contribution of CIN on prognosis has been generally lacking. Some have attempted to link gene expression signatures prevalent in aneuploid cells to patient survival and prognosis (71), using aneuploidy as a surrogate marker for CIN. Some of the aneuploidy-related gene expression signatures that correlated with poor prognosis were indeed involved in the maintenance of microtubule stability, such as the expression of TPX2 (73). Interestingly, it was also found that, in many cancer types, deregulation of the genetic signature associated with CIN generally led to reduced patient survival and inferior outcome; however, gene expression signatures at the extreme end of the spectrum paradoxically had improved survival, and their tumors responded more readily to therapy (74). Furthermore, overexpression of cyclin E (with concomitant increase in heterogeneous aneuploidy) correlated with poor prognosis and inferior outcome in patients diagnosed with DLBCL (75). Finally, in a similar cohort of patients diagnosed with DLBCL, work directly examining chromosome missegregation events in cells fixed while undergoing anaphase showed that a two-fold increase in chromosome missegregation frequency significantly correlated with overall survival, prognosis, tumor stage, and treatment response (48). Furthermore, in these patients, a two-fold increase in the rates of chromosome missegregation significantly correlated with the tumor's ability to spread to distant sites, including the bone marrow. This preliminary evidence is encouraging; however, a firm understanding of the role of CIN on tumor prognosis is still lacking in most cancer types.

\section{CIN as a therapeutic target}

Therapeutic targeting of CIN in cancer is still at its preclinical stages. Nevertheless, kinetochores represent an attractive therapeutic target to modify chromosome missegregation in cancer, given their role in regulating microtubule attachments to chromosomes $(20,30,38,76,77)$. Overexpression of kinetochore proteins that destabilize attached microtubules is sufficient to significantly decrease chromosome missegregation rates and suppress CIN $(29,30)$. Alternatively, increasing microtubule stability (for instance, by using inhibitors of the microtubule destabilizing kinase, Aurora B; ref. 78) increases chromosome missegregation rates. In fact, these inhibitors have shown preliminary success in treating primary as well as resistant tumors (79-82). However, a common dose-limiting adverse effect of many new mitotic drugs is severe bone marrow toxicity and subsequent neutropenia (82). A possible approach to circumvent this toxicity would be to target CIN as an adjuvant therapy to standard treatment, thus limiting the ability of tumors to acquire drug resistance and relapse.

The nature of therapeutic interventions targeting CIN will inevitably depend on the existing levels of chromosome missegregation in the specific tumor as well as the effect of either 
decreasing or increasing chromosome missegregation on tumor prognosis. In tumors in which CIN is associated with poor prognosis, such as DLBCL (48), suppressing chromosome missegregation could reduce the frequency of metastasis and drug resistance. Yet, given that extremely elevated rates of CIN also appear to decrease tumor fitness in some cancers $(67,74,83)$, further increasing chromosome missegregation rates could be beneficial. Therefore, determining chromosome missegregation frequencies of a given tumor and comparing these to the optimal level of CIN that allows for tumor adaptation might be a prerequisite to any therapeutic approach targeting CIN.

\section{Concluding remarks}

In summary, it is evident that chromosome missegregation may be as important a factor in tumor development as DNA mutations and chromosomal translocations. Until recently, little attention has been paid to the process of whole-chromosome imbalance. Given the widespread relevance of CIN in human cancers, understanding the mechanisms that lead to chromosome missegregation, the role it plays in the evolution of tumors, and the potential for therapeutic intervention will provide a significant improvement in our ability to find cures for resistant cancers.

Note added in proof. Recent work demonstrates that chromosomal instability can lead to DNA breaks and chromosome pulverization by the formation of micronuclei that replicate out of phase from the main cellular nucleus (84).

\section{Acknowledgments}

We thank Ashley M. Laughney (Dartmouth College, Hanover, New Hampshire, USA) for technical help with the figures and Peter Cuadrilla for critical feedback. S.F. Bakhoum is supported by the Hitchcock-Foundation grant 250-4041, and D.A. Compton is supported by the NIH grant GM51542.

Address correspondence to: Samuel F. Bakhoum, Department of Biochemistry and Norris Cotton Cancer Center, HB7200, Dartmouth Medical School, Hanover, New Hampshire 03755, U.S.A. Phone: 603.276.9791; Fax: 603.650.1128; E-mail: samuel. bakhoum@dartmouth.edu.
1. Nowell PC. Clonal evolution of tumor-cell populations. Science. 1976;194(4260):23-28.

2. Lengauer C, Kinzler KW, Vogelstein B. Genetic instability in colorectal cancers. Nature. 1997; 386(6625):623-627.

3. Lengauer C, Kinzler KW, Vogelstein B. Genetic instabilities in human cancers. Nature. 1998; 396(6712):643-649.

4. Cimini D. Merotelic kinetochore orientation, aneuploidy, and cancer. Biochim Biophys Acta. 2008; 1786(1):32-40.

5. Thompson SL, Compton DA. Examining the link between chromosomal instability and aneuploidy in human cells. J Cell Biol. 2008;180(4):665-672.

6 . Boveri $\mathrm{T}$. Concerning the origin of malignant tumours by Theodor Boveri. Translated and annotated by Henry Harris. J Cell Sci. 2008;121 suppl 1:1-84.

7. Receveur A, et al. Trisomy 4 associated with double minute chromosomes and MYC amplification in acute myeloblastic leukemia. Ann Genet. 2004; 47(4):423-427.

8. Goldstone A, et al. The importance of diagnostic cytogenetics on outcome in AML: Analysis of 1,612 patients entered into the MRC AML 10 trial. Blood. 1998;92(7):2322-2333.

9. Grimwade D, et al. Hierarchical cytogenetic classification is highly predictive of outcome in AML arising at all ages: Analysis of 4225 cases entered in the UK MRC leukaemia trials. Blood. 2000;96:825a

10. Gulley ML, Shea TC, Fedoriw Y. Genetic tests to evaluate prognosis and predict therapeutic response in acute myeloid leukemia. J Mol Diagn. 2010; 12(1):3-16

11. Kobayashi K, Usami I, Kubota M, Nishio T, Kakazu N. Chromosome 7 abnormalities in acute megakaryoblastic leukemia associated with Down syndrome. Cancer Genet Cytogenet. 2005;158(2):184-187.

12. Sullivan SG, Hussain R, Glasson EJ, Bittles AH. The profile and incidence of cancer in Down syndrome. J Intellect Disabil Res. 2007;51(pt 3):228-231.

13. Patja K, Pukkala E, Sund R, Iivanainen M, Kaski M. Cancer incidence of persons with Down syndrome in Finland: A population-based study. Int J Cancer. 2006;118(7):1769-1772.

14. Hunter A. High risk of malignancy in mosaic variegated aneuploidy syndrome. Am J Med Genet A. 2003; $117 \mathrm{~A}(2): 199$.

15. Jacquemont S, Boceno M, Rival JM, Mechinaud F, David A. High risk of malignancy in mosaic variegated aneuploidy syndrome. Am J Med Genet. 2002;109(1):17-21.
16. Rahman N, et al. Constitutional aneuploidy and cancer predisposition caused by biallelic mutations in BUB1B. Nat Genet. 2004;36(11):1159-1161.

17. Paulsson K, Johansson B. Trisomy 8 as the sole chromosomal aberration in acute myeloid leukemia and myelodysplastic syndromes. Pathol Biol (Paris). 2007;55(1):37-48.

18. Compton DA. Spindle assembly in animal cells. Annu Rev Biochem. 2000;69:95-114.

19. Maiato H, DeLuca J, Salmon ED, Earnshaw WC. The dynamic kinetochore-microtubule interface. J Cell Sci. 2004;117(pt 23):5461-5477.

20. Thompson SL, Bakhoum SF, Compton DA. Mechanisms of chromosomal instability. Curr Biol. 2010;20(6):R285-R295.

21. Pati D, et al. Overexpression of Separase induces aneuploidy and mammary tumorigenesis. Proc Natl Acad Sci U S A. 2008;105(35):13033-13038.

22. Di Leonardo A, Lentini L, Amato A, Schillaci T. Simultaneous Aurora-A/STK15 overexpression and centrosome amplification induce chromosomal instability in tumour cells with a MIN phenotype. BMC Cancer. 2007;7:212.

23. Silkworth WT, Nardi IK, Scholl LM, Cimini D. Multipolar spindle pole coalescence is a major source of kinetochore mis-attachment and chromosome mis-segregation in cancer cells. PloS One. 2009; 4(8):e6564.

24. Ganem NJ, Godinho SA, Pellman D. A mechanism linking extra centrosomes to chromosomal instability. Nature. 2009;460(7252):278-282.

25. Tusell L, Pampalona J, Soler D, Frias C, Genesca A. Different outcomes of telomere-dependent anaphase bridges. Biochem Soc Trans. 2010;38(6):1698-1703.

26. Cahill DP, et al. Mutations of mitotic checkpoint genes in human cancers. Nature. 1998; 392(6673):300-303.

27. Sotillo R, Schvartzman JM, Socci ND, Benezra R. Mad2-induced chromosome instability leads to lung tumour relapse after oncogene withdrawal. Nature. 2010;464(7287):436-440.

28. Diaz-Rodriguez E, Sotillo R, Schvartzman JM, Benezra R. Hec1 overexpression hyperactivates the mitotic checkpoint and induces tumor formation in vivo. Proc Natl Acad Sci U S A. 2008;105(43):16719-16724.

29. Bakhoum SF, Thompson SL, Manning AL, Compton DA. Genome stability is ensured by temporal control of kinetochore-microtubule dynamics. Nat Cell Biol. 2009;11(1):27-35.

30. Bakhoum SF, Genovese G, Compton DA. Deviant kinetochore microtubule dynamics under- lie chromosomal instability. Curr Biol. 2009; 19(22):1937-1942.

31. Cimini D, Howell BJ, Degrassi F, Salmon ED. Merotelic attachment of single kinetochores to microtubules from opposite poles is not detected by the mitotic spindle checkpoint and induces chromosome loss during mitosis. Mol Biol Cell. 2000; 11:430a.

32. Cimini D, Howell B, Maddox P, Khodjakov A, Degrassi F, Salmon ED. Merotelic kinetochore orientation is a major mechanism of aneuploidy in mitotic mammalian tissue cells. J Cell Biol. 2001; 153(3):517-527.

33. Khodjakov A, Magidson V, O'Connell CB, Loncarek J, Paul R, Mogilner A. The spatial arrangement of chromosomes during prometaphase facilitates spindle assembly. Cell. 2011;146(4):555-567.

34. Kapoor TM, et al. Chromosomes can congress to the metaphase plate before biorientation. Science. 2006;311(5759):388-391.

35 . Loncarek J, et al. The centromere geometry essential for keeping mitosis error free is controlled by spindle forces. Nature. 2007;450(7170):745-749.

36. Cheeseman IM, Desai A. Molecular architecture of the kinetochore-microtubule interface. Nat Rev Mol Cell Biol. 2008;9(1):33-46.

37. Desai A, Mitchison TJ. Microtubule polymerization dynamics. Annu Rev Cell Dev Biol. 1997;13:83-117.

38. DeLuca JG, Gall WE, Ciferri C, Cimini D, Musacchio A, Salmon ED. Kinetochore microtubule dynamics and attachment stability are regulated by Hec1. Cell. 2006;127(5):969-982.

39. Bakhoum SF, Compton DA. Cancer: CINful centrosomes. Curr Biol. 2009;19(15):R642-R645.

40. Sasaki K, et al. Overexpression of BUBR1 is associated with chromosomal instability in bladder cancer. Cancer Genet Cytogenet. 2007;174(1):42-47.

41. Michel LS, et al. MAD2 haplo-insufficiency causes premature anaphase and chromosome instability in mammalian cells. Nature. 2001;409(6818):355-359.

42. Lampson MA, Kapoor TM. The human mitotic checkpoint protein BubR1 regulates chromosomespindle attachments. Nat Cell Biol. 2005;7(1):93-98.

43. Musacchio A, Salmon ED. The spindle-assembly checkpoint in space and time. Nat Rev Mol Cell Biol. 2007;8(5):379-393.

44. Manning AL, Bakhoum SF, Maffini S, CorreiaMelo C, Maiato H, Compton DA. CLASP1, astrin and Kif2 $\mathrm{b}$ form a molecular switch that regulates kinetochore-microtubule dynamics to promote mitotic progression and fidelity. EMBO J. 2010; 
29(20):3531-3543.

45. Fujiwara T, Bandi M, Nitta M, Ivanova EV, Bronson RT, Pellman D. Cytokinesis failure generating tetraploids promotes tumorigenesis in p53-null cells. Nature. 2005;437(7061):1043-1047.

46. Campbell PJ, et al. Massive genomic rearrangement acquired in a single catastrophic event during cancer development. Cell. 2011;144(1):27-40.

47. Meyerson M, Pellman D. Cancer genomes evolve by pulverizing single chromosomes. Cell. 2011;144(1):9-10.

48. Bakhoum SF, Danilova OV, Kaur P, Levy NB, Compton DA. Chromosomal instability substantiates poor prognosis in patients with diffuse large B-cell lymphoma. Clin Cancer Res. 2011;17(24):7704-7711.

49. Mitelman F, Johansson B, Mertens F, eds. Mitelman Database of Chromosome Aberrations and Gene Fusions in Cancer. NIH Web site. http://cgap. nci.nih.gov/Chromosomes/Mitelman. Updated November 21, 2011. Accessed February 13, 2012.

50. Walther A, Houlston R, Tomlinson I. Association between chromosomal instability and prognosis in colorectal cancer: a meta-analysis. Gut. 2008;57(7):941-950.

51. Florl AR, Schulz WA. Chromosomal instability in bladder cancer. Arch Toxicol. 2008;82(3):173-182.

52. M'kacher R, et al. JC human polyomavirus is associated to chromosomal instability in peripheral blood lymphocytes of Hodgkin's lymphoma patients and poor clinical outcome. Ann Oncol. 2010; 21(4):826-832.

53. Knudson CM, van de Wetering CI. Chromosomal instability and supernumerary centrosomes represent precursor defects in a mouse model of T-cell lymphoma. Cancer Res. 2007;67(17):8081-8088.

54. Kramer A, et al. Centrosome aberrations as a possible mechanism for chromosomal instability in non-Hodgkin's lymphoma. Lenkemia. 2003; 17(11):2207-2213.

55. Schvartzman JM, Sotillo R, Benezra R. Mitotic chromosomal instability and cancer: mouse modelling of the human disease. Nat Rev Cancer. 2010; 10(2):102-115

56. Weaver BA, Silk AD, Montagna C, Verdier-Pinard P, Cleveland DW. Aneuploidy acts both oncogenically and as a tumor suppressor. Cancer Cell. 2007; 11(1):25-36.

57. Schvartzman JM, Duijf PH, Sotillo R, Coker C, Benezra R. Mad2 is a critical mediator of the chromosome instability observed upon $\mathrm{Rb}$ and $\mathrm{p} 53$ pathway inhibition. Cancer Cell. 2011;19(6):701-714.

58. Thompson SL, Compton DA. Proliferation of aneuploid human cells is limited by a p53-depen- dent mechanism. J Cell Biol. 2010;188(3):369-381.

59. Fragouli E, et al. Transcriptomic profiling of human oocytes: association of meiotic aneuploidy and altered oocyte gene expression. Mol Hum Reprod. 2010;16(8):570-582

60. Yaspo ML, et al. Transcript level alterations reflect gene dosage effects across multiple tissues in a mouse model of down syndrome. Genome Res. 2004; 14(7):1258-1267.

61. Baker DJ, Jin F, Jeganathan KB, van Deursen JM. Whole chromosome instability caused by Bub1 insufficiency drives tumorigenesis through tumor suppressor gene loss of heterozygosity. Cancer Cell. 2009;16(6):475-486.

62. Janssen A, van der Burg M, Szuhai K, Kops GJPL, Medema RH. Chromosome segregation errors as a cause of DNA damage and structural chromosome aberrations. Science. 2011;333(6051):1895-1898.

63. Weaver BA, Cleveland DW. The aneuploidy paradox in cell growth and tumorigenesis. Cancer Cell. 2008;14(6):431-433.

64. Janssen A, Kops GJ, Medema RH. Elevating the frequency of chromosome mis-segregation as a strategy to kill tumor cells. Proc Natl Acad Sci U S A. 2009; 106(45):19108-19113.

65. Galimberti F, et al. Targeting the cyclin E-Cdk-2 complex represses lung cancer growth by triggering anaphase catastrophe. Clin Cancer Res. 2010; 16(1):109-120.

66. Rao CV, et al. Colonic tumorigenesis in BubR1(+/-) $\operatorname{Apc}(\mathrm{Min} /+)$ compound mutant mice is linked to premature separation of sister chromatids and enhanced genomic instability. Proc Natl Acad SciUS A. 2005;102(12):4365-4370.

67. Komarova NL, Wodarz D. The optimal rate of chromosome loss for the inactivation of tumor suppressor genes in cancer. Proc Natl Acad Sci US A. 2004;101(18):7017-7021.

68. Andersson SGE, Kurland CG. Reductive evolution of resident genomes. Trends Microbiol. 1998; 6(7):263-268.

69. Weaver BA, Silk AD, Cleveland DW. Low rates of aneuploidy promote tumorigenesis while high rates of aneuploidy cause cell death and tumor suppression. Cell Oncol. 2008;30(5):453.

70. Smid M, et al. Patterns and incidence of chromosomal instability and their prognostic relevance in breast cancer subtypes. Breast Cancer Res Treat. 2011; 128(1):23-30.

71. Szallasi Z, Carter SL, Eklund AC, Kohane IS, Harris LN. A signature of chromosomal instability inferred from gene expression profiles predicts clinical outcome in multiple human cancers. Nat
Genet. 2006;38(9):1043-1048.

72. Li R, et al. Aneuploidy confers quantitative proteome changes and phenotypic variation in budding yeast. Nature. 2010;468(7321):321-325.

73. Guarguaglini G, Asteriti IA, Rensen WM, Lindon C, Lavia P. The Aurora-A/TPX2 complex: A novel oncogenic holoenzyme? Biochim Biophys Acta. 2010; 1806(2):230-239.

74. Birkbak NJ, et al. Paradoxical relationship between chromosomal instability and survival outcome in cancer. Cancer Res. 2011;71(10):3447-3452.

75. Tzankov A, et al. Diffuse large B-cell lymphoma with overexpression of cyclin E substantiates poor standard treatment response and inferior outcome. Clin Cancer Res. 2006;12(7 pt 1):2125-2132.

76. Cheeseman IM, Chappie JS, Wilson-Kubalek EM, Desai A. The conserved KMN network constitutes the core microtubule-binding site of the kinetochore. Cell. 2006;127(5):983-997.

77. Cimini D, Wan XH, Hirel CB, Salmon ED. Aurora kinase promotes turnover of kinetochore microtubules to reduce chromosome segregation errors. Curr Biol. 2006;16(17):1711-1718.

78. Liu D, Vader G, Vromans MJ, Lampson MA, Lens SM. Sensing chromosome bi-orientation by spatial separation of aurora B kinase from kinetochore substrates. Science. 2009;323(5919):1350-1353.

79. Mahadevan D, Liu X, Persky DO, Miller TP, Squires MS, Wenqing Q. AT9283, a novel pan- Aurora/ JAK-2 kinase inhibitor suppresses tumor growth in aggressive B-cell non-Hodgkin's lymphoma [abstract]. Blood. 2010;116:3930.

80. Schellens JH, et al. Clinical evaluation of AZD1152, an i.v. inhibitor of Aurora B kinase, in patients with solid malignant tumors. Ann Oncol. 2011; 22(2):431-437.

81. Payton M, et al. Preclinical evaluation of AMG 900 , a novel potent and highly selective panaurora kinase inhibitor with activity in taxane-resistant tumor cell lines. Cancer Res. 2010; 70(23):9846-9854.

82. Tsuboi K, et al. A Phase I study to assess the safety, pharmacokinetics and efficacy of barasertib (AZD1152), an Aurora B kinase inhibitor, in Japanese patients with advanced acute myeloid leukemia. Leuk Res. 2011;35(10):1384-1389.

83. Roylance R, et al. Relationship of extreme chromosomal instability with long-term survival in a retrospective analysis of primary breast cancer. Cancer Epidemiol Biomarkers Prev. 2011;20(10):2183-2194.

84. Crasta K, et al. DNA breaks and chromosome pulverization from errors in mitosis. Nature. 2012; 482(7383):53-58 